\title{
Anterior Segment Optical Coherence Tomography of Ocular Cystinosis Confirmed by Electron Microscopy
}

\author{
Abhinav Golla Mitchell P. Weikert Patricia Chevez-Barrios Zaina Al-Mohtaseb \\ Cullen Eye Institute, Department of Ophthalmology, Baylor College of Medicine, Houston, TX, USA
}

\section{Established Facts}

- Ocular cystinosis is thought to have characteristic crystalline deposits at least as deep as the anterior stroma.

\section{Novel Insights}

- To our knowledge, this is the first reported case of ocular cystinosis confirmed by pathology that involves strictly the epithelial layer of the cornea and has been imaged by anterior segment optical coherence tomography.

\section{Keywords}

Anterior segment optical coherence tomography · Ocular cystinosis · Electron microscopy

\footnotetext{
Abstract

Purpose: To report a case of ocular cystinosis diagnosed by anterior segment optical coherence tomography (AS-OCT) and histopathology. Methods: A 67-year-old man who presented with eye pain was found to have unilateral corneal crystal deposition. Ocular cystinosis was diagnosed by histopathology, AS-OCT, and lack of renal involvement of the disease. Results: AS-OCT showed hyperreflective densities in the corneal epithelium. The patient underwent superficial keratectomy given irregular astigmatism prior to cataract
}

\section{KARGER}

(c) 2018 S. Karger AG, Basel

E-Mail karger@karger.com

www.karger.com/oop surgery. Electron microscopy of the corneal scrapings revealed epithelial cells with intracystoplasmic membranebound crystals confirming the diagnosis of cystinosis. Conclusion: To our knowledge, this is the first reported case of ocular cystinosis involving strictly the epithelial layer of the cornea that has been imaged by AS-OCT.

(C) 2018 S. Karger AG, Basel

\section{Introduction}

Cystinosis is a rare, autosomal recessive mutation in the CTNS gene on chromosome $17 \mathrm{p} 13$ that can lead to intracellular accumulation of cystine crystals in the eyes, kidneys, bone marrow, liver, spleen, thyroid, pancreas,

Zaina Al-Mohtaseb, MD

Department of Ophthalmology, Baylor College of Medicine 6565 Fannin, NC205

Houston, TX 77030 (USA)

E-Mail zaina@bcm.edu 

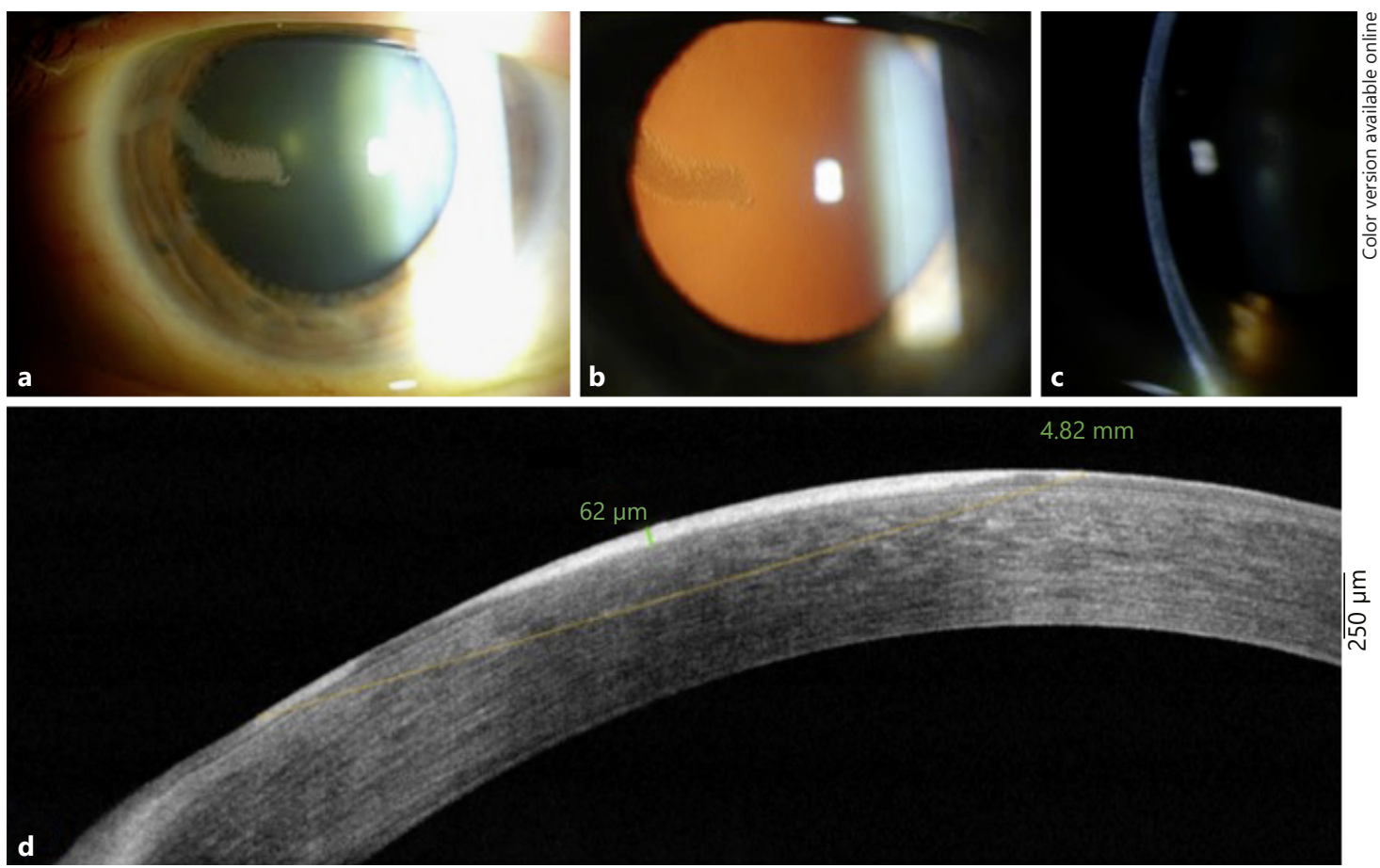

Fig. 1. a Slit-lamp photo: subepithelial crystalline deposits that extend from the 9:30 position on the limbus to the central cornea with a 1.55 -mm-wide band. They do not penetrate Bowman's membrane. b Retro-illumination. c Oblique slit lamp. d Anterior segment OCT: hyperreflectivity of uniform density with a thickness of 62 $\mu \mathrm{m}$, spanning about $4.82 \mathrm{~mm}$ and tapering near the periphery of the lesion with very sharp margins.

and muscles [1]. This can result in photophobia, hepatosplenomegaly, hypothyroidism, diabetes, and muscle weakness. There are three main types of cystinosis, one of which is ocular cystinosis, characterized by corneal and conjunctival crystal deposition and no renal involvement. Cystinosis typically presents as one of three clinical forms: infantile nephropathic (95\%), juvenile (5\%), and adult/ non-nephropathic. The adult cystinosis typically spares the kidney and other organs and is restricted to only ocular findings. The three forms of cystinosis can essentially be distinguished by age of onset and severity of renal disease. Here we present a case of ocular cystinosis diagnosed by slit-lamp biomicroscopy, anterior segment optical coherence tomography (AS-OCT), and transmission electron microscopy (TEM), which demonstrated pathognomonic intracellular crystalline deposits in the corneal epithelium.

The rate of corneal crystal accumulation is correlated to the severity of the CTNS mutation [2]. Therefore, a patient's Corneal Cystine Crystal Score (calculated from 0-3 based on opacity, density of crystals, and number of crystals) is reflective of the severity of their disease.

AS-OCT of Ocular Cystinosis Confirmed by Electron Microscopy
AS-OCT images were obtained using an Avanti (Optovue, Inc.) Widefield Enface Spectral-Domain OCT with $70,000 \mathrm{~A}-\mathrm{Scan} / \mathrm{s}$, axial resolution $5 \mu \mathrm{m}$, and transverse resolution $15 \mu \mathrm{m}$.

\section{Case Report}

A 67-year-old male presented to his ophthalmologist with 6 months of ache behind the left eye. The patient denied any recent changes in vision, photophobia, flashes, floaters, or trauma. He had no history of eye surgery or trauma.

The patient had a corrected visual acuity of $20 / 25$ in the right eye and 20/40 in the left. Intraocular pressures were 16 and $14 \mathrm{~mm}$ $\mathrm{Hg}$ in the right and left eye, respectively. Both pupils were equal and reactive without a relative afferent pupillary defect, and extraocular movements were full.

On slit-lamp exam, the left cornea had subepithelial crystalline deposits that extended from the 9:30 position on the limbus to the central cornea in a band about $1.5 \mathrm{~mm}$ wide. They did not penetrate Bowman's layer. There was no associated corneal thinning or edema. The anterior chamber was deep and quiet bilaterally. There were bilateral $2+$ nuclear sclerotic cataracts and posterior vitreous detachments, and a normal fundus exam with no evidence of retinopathy or retinal crystalline deposits. 
Fig. 2. a Corneal epithelium seen under polarization displays intracytoplasmic retractile crystals. Hematoxylin and eosin stain; $100 \%$ alcohol fixation and processing. Original magnification: $40 \times$. b Transmission electron microscopy from the alcoholfixed paraffin-embedded corneal epithelium shows intracytoplasmic membranebound crystals typical of those of cystinosis. Direct magnification: $30,000 \times$.
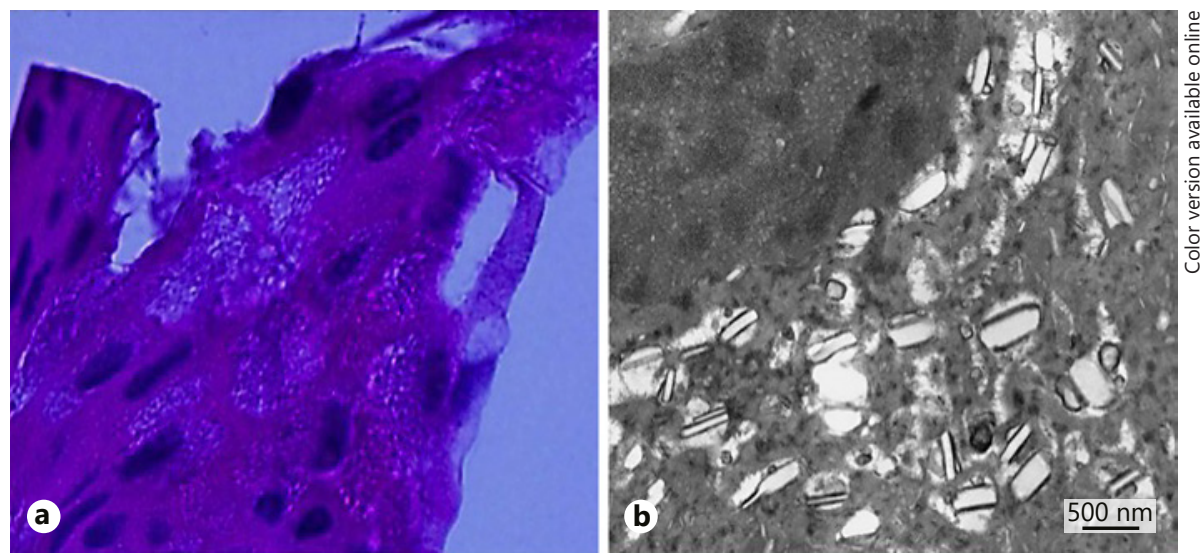

AS-OCT showed an epithelial hyperreflectivity of uniform density with a thickness of $62 \mu \mathrm{m}$, spanning about $4.82 \mathrm{~mm}$ and tapering near the periphery of the lesion with very sharp margins (Fig. 1).

TEM analysis of a corneal biopsy specimen confirmed the diagnosis of ocular cystinosis by demonstrating epithelial cells with intracytoplasmic crystalline deposits. These deposits stained negatively with PAS (glycoproteins/mucin), von Kossa (calcium), and trichrome Masson's (hyaline deposits) (Fig. 2).

\section{Discussion}

To our knowledge, this is the first reported case of ocular cystinosis involving strictly the epithelial layer of the cornea that has been imaged by AS-OCT. Previous reports of cystinosis using OCT have been described, with similar hyperreflective deposits observed predominantly within the anterior stroma [3]. However, our findings showed a crystalline deposit depth of only $62 \mu \mathrm{m}$, which contrasted with the results of Labbe et al. [3] reporting a mean depth of $291.40 \pm 81.42 \mu \mathrm{m}$. Ocular cystinosis can manifest as crystals in any layer of the cornea, with a study showing that the highest density of crystals was found in anterior stroma, followed by Bowman's layer and the middle stroma [3]. It is unclear why the disease in this case report was unilateral and well demarcated, as there are no other reports of unilateral or well-demarcated ocular cystinosis to our knowledge in the literature. Potentially the crystal concentration and location will develop over time. Also, there could have been a reactive inflammatory process that took place that unmasked the cystinosis in the damaged cells.

The TEM results from our case report were comparable to those of Wong et al. [4] and Stefani and Vogel [5], where they described intracellular cystine crystals within conjunctival fibroblasts and macrophages.
In addition to OCT and TEM, other studies also used confocal microscopy, describing cystinosis crystals as spindle- or needle-shaped bodies measuring from 1 to $175 \mu \mathrm{m}$ in length and 1 to $30 \mu \mathrm{m}$ in thickness [3]. However, the patients in these studies had nephropathic cystinosis, not the milder ocular cystinosis presented in our case report. In a case report by Kocabora et al. [6], these crystals were described as variable in size and shape, being square, oblong, or rhomboid at $0.5-2 \mu \mathrm{m}$ in length. This is comparable to the pathology specimen from our case report, which showed oblong crystals of varying size on TEM.

The symptoms and exam findings of cystinosis are quite unique; however, it is helpful to rule out crystalline keratopathy due to multiple myeloma, other immunoglobulin abnormalities, or medication-induced corneal deposits [7]. Confocal microscopy, TEM, and OCT can all be used to aid in differentiating crystalline keratopathies, such as Bietti's, Schnyder's, or infectious crystalline corneal dystrophy [7]. Bietti's corneal dystrophy has superficial punctiform corneal crystals, whereas ocular cystinosis has needle-shaped corneal crystals. Additionally, nearly all patients with Bietti's develop retinal involvement with visual loss [8]. Schnyder's corneal dystrophy typically presents with glare from the corneal haze, while ocular cystinosis typically presents with photophobia. Additionally, ocular cystinosis typically has crystals in the anterior peripheral corneal stroma, whereas Schnyder's usually presents as central panstromal haze with arcus lipoides [8]. Infectious crystalline corneal dystrophy is an acquired disease that occurs typically after a corneal transplant, whereas ocular cystinosis is an autosomal dominant inherited disease [8]. This was a case of classic ocular cystinosis with no renal findings, and it can be differenti- 
ated from other similar corneal dystrophies based on the needle-shaped crystals, location of the crystals, and history of photophobia. Our TEM results were consistent with ocular cystinosis. Unfortunately, our patient did not have medical insurance and did not want to pay out of pocket for any extra tests. According to him, prior to losing his insurance, he had normal kidney function, though. Our inability to perform a systemic workup is a weakness of this case report.

In conclusion, the use of AS-OCT to describe the pathology of ocular cystinosis is critical to further our understanding of this disease.

\section{Statement of Ethics}

This case report complies with the guidelines for human studies and animal welfare regulations. For this case report, the subject was given their informed consent. No IRB was necessary as a research study was not conducted.

\section{Disclosure Statement}

No author has a financial or proprietary interest in any material or method mentioned. There was no sponsorship or funding arrangements relating to the research for any author. No author has any conflict of interest.

\section{References}

1 Kalatzis V, Nevo N, Cherqui S, Gasnier B, Antignac C: Molecular pathogenesis of cystinosis: effect of CTNS mutations on the transport activity and subcellular localization of cystinosin. Hum Mol Genet 2004;13: 1361-1371.

2 Gahl WA, Kuehl EM, Iwata F, et al: Corneal crystals in nephropathic cystinosis: natural history and treatment with cysteamine eyedrops. Mol Genet Metab 2000;71:100-120.
3 Labbe A, Niaudet P, Loirat C, Charbit M, Guest G, Baudouin C: In vivo confocal microscopy and anterior segment optical coherence tomography analysis of the cornea in nephropathic cystinosis. Ophthalmology 2009; 116:870-876.

4 Wong VG, Kuwabara T, Brubaker R, Olson W, Schulman J, Seegmiller JE: Intralysosomal cysteine crystals in cystinosis. Invest Ophthalmol 1970;9:83-88.

5 Stefani FH, Vogel S: Adult cystinosis: electron microscopy of the conjunctiva. Graefes Arch Clin Exp Ophthalmol 1982;219:143-145.
6 Kocabora MS, Ozbilen KT, Altunsoy M, Ahishali B, Taskapili M: Clinicopathological features of ocular cystinosis. Clin Exp Ophthalmol 2008;36:778-781.

7 Kleta R, Blair SC, Bernardini I, Kaiser-Kupfer MI, Gahl WA: Keratopathy of multiple myeloma masquerading as corneal crystals of ocular cystinosis. Mayo Clin Proc 2004;79: 410-412.

8 Weiss JS, Kemichian AJ: Differential diagnosis of Schnyder corneal dystrophy. Dev Ophthalmol 2011;48:67-96. 